\title{
Perfiles de comportamiento de los padres en el deporte y su re- lacíon con los procesos motivacionales de sus hijos
}

\author{
Profiles of parental behavior in sport and its relationship with their chil- \\ dren' motivational processes
}

Pedro Antonio Sánchez-Miguel ${ }^{1}$, Juan José Pulido González², Diana Amado Alonso ${ }^{3}$,
Francisco Miguel Leo Marcos ${ }^{1}$, David Sánchez-Oliva², Inmaculada González Ponce ${ }^{2}$
ARTIGO ORIGINAL | ORIGINALARTICLE

RESUMEN

El principal objetivo del estudio fue examinar perfiles de padres respecto a la presión/implicación y apoyo a las necesidades psicológicas básicas (NPB) sobre la motivación y aburrimiento desarrollado por jóvenes deportistas. La muestra estuvo formada por 301 padres y sus respectivos 301 hijos ( $\mathrm{M}=$ 13.92; DT $=2.04)$, quiénes pertenecían a deportes colectivos $(n=191)$ e individuales $(n=128)$. Se midieron en padres la percepción de implicación y presión, al igual que el apoyo a las NPB, mientras que la motivación autodeterminada y el aburrimiento en la práctica fueron variables valoradas en adolescentes. Los resultados muestran la existencia de dos perfiles (perfil adaptativo, que estaba compuesto por el apoyo a las NPB y bajos niveles de presión, y perfil desadaptativo, que estaba formado por altos niveles de presión y bajos valores de apoyo a las NPB), y una relación entre el perfil desataptativo de los padres y la motivación de los niños y su aburrimiento en la práctica. Asimismo, se demostró que los padres con un perfil adaptativo realizaban menos actividad física durante la semana. Para concluir, se destaca la importancia de que los padres generen bajos niveles de presión y alta implicación en la práctica deportiva de sus hijos, así como promover el apoyo a las NPB, así como incrementar sus niveles de actividad física, con el objetivo de mejorar la motivación de los jóvenes deportistas y descender los niveles de aburrimiento en la práctica.

Palavras-clave: Teoría de la Autodeterminación, padres, deporte escolar, jóvenes deportistas.

\begin{abstract}
The main aim of the study was to examine parents' profiles respecting pressure/involvement and support to the basic psychological needs (BPN) on motivation and boredom developed by youth participants in sport practice. The sample was formed by 301 parents and their respective 301 children $(\mathrm{M}=$ 13.92; SD $=2.04)$, who belonged to team sports $(n=191)$ and individual sports $(n=128)$. Perception of involvement and pressure, as well as support to the BPN were measure in parents, whereas self-determined motivation and boredom in the practice were variables assessed in adolescents. Results showed the existence of two profiles (adaptive profile, which was composed of support to the BPN and low levels of pressure, and maladaptive profile, which was formed by high level of pressure and low levels of support to the BPN), and a relationship between parents' maladaptive profile and children's motivation and boredom. Moreover, it was revealed that parents with a lower adaptive profile showed less physical activity during the week. To conclude, it is highlighted the importance to create a lower pressure and greater involvement in the adequate practice of their children, as well as promote support to the BPN, and increase their level of physical activity, with the aim to enhance youth athletes' motivation and decrease their boredom levels in the practice.

Keywords: Self-Determination Theory, parents, scholar sport, youth athletes
\end{abstract}

\author{
Artigo recebido a 18.04.2014; Aceite a 07.10.2014 \\ ${ }^{1}$ Faculty of Teaching Training. Department of Didactics of Musical, Plastic and Corporal Expression. Univer- \\ sity of Extremadura \\ ${ }^{2}$ Faculty of Sports Sciences. Department of Didactics of Musical, Plastic and Corporal Expression. University \\ of Extremadura \\ ${ }^{3}$ UCAM. Catholic University of Murcia. Faculty of Physical Activity and Sports Sciences \\ * Autor correspondente: Dr. Pedro Antonio Sánchez Miguel. Facultad de Formación del Profesorado. Avenida \\ de la Universidad, S/N, 10003, Cáceres.E-mail: pesanchezm@unex.es
}




\section{INTRODUCCIÓN}

El fomento de la actividad física y el deporte en edades tempranas es un objetivo a alcanzar por todos los significativos (padres, entrenadores y compañeros) que están en contacto con el joven deportista (Holt, Tamminen, Black, Mandigo, \& Fox, 2009). En este sentido, la práctica de actividad física y el apoyo por parte de los padres representa una de las formas de influencia social más analizada en adolescentes (Edwardson \& Gorely, 2010), incidiendo en gran medida en las consecuencias que puede derivar la práctica en el contexto deportivo (Sánchez-Miguel, Leo, Sánchez-Oliva, Amado, \& García-Calvo, 2013).

La mayoría de los trabajos que han analizado la relación entre padres e hijos, sostienen la idea de que aquellos entornos donde exista un control psicológico por parte de los padres (e.g.: Comunicación de reglas sin valorar la perspectiva de los niños, inconsistencia en las normas establecidas, presión hacia ellos, etc.) conducen hacia consecuencias psicológicas negativas en los hijos, como son la falta de motivación, aburrimiento, falta de compromiso, etc. (Soenens \& Vansteenkiste, 2010). Este control psicológico hace referencia a comportamientos donde se introduce a los jóvenes en pensamientos negativos mediante el uso de técnicas manipulativas, como avergonzar, ejercer una gran presión, no implicarse en las actividades que éstos realizan, etc., que conllevan consecuencias conductuales tanto a nivel global en los jóvenes (trabajo, esfuerzo, respeto, tolerancia, etc) y a nivel deportivo en variables como el abandono (Sánchez-Miguel, 2010), atrapamiento, ansiedad competitiva, etc. (Steinberg, 2005). Por el contrario, hay un consenso general en la comunidad científica que indica que los entornos de apoyo hacia la autonomía, competencia y fomento de las relaciones sociales por parte de los padres, generan multitud de beneficios en los adolescentes y favorece el paso hacia la etapa adulta (Sheldon \& Filak, 2008).

En relación a esas dos fases de control y fomento del apoyo por parte de los padres, surgen variables como la presión que los padres puedan ejercer en la actividad deportiva y la implicación en la misma. La percepción de presión hace referencia a la situación dónde los padres empujan a sus hijos a competir duro y/o ganar y cuando el afecto hacia sus hijos puede estar condicionado por la participación deportiva y/o los resultados, mientras que la percepción de implicación de los padres hace referencia a la participación de éstos en el deporte, compartiendo su tiempo, dinero e intereses (Holt et al., 2009).

Estas variables de presión e implicación pueden influir en la adopción de comportamientos adaptativos en contextos sociales como la motivación (Deci \& Ryan, 2000). En referencia a ello, una de las teorías de la motivación que más está analizando la motivación de los individuos en contextos sociales es la Teoría de la Autodeterminación (TAD: Deci \& Ryan, 2000; Ryan \& Deci, 2000). La TAD defiende que una persona es un ente activo con tendencia a la superación personal y al desarrollo psicológico, que evoluciona tratando de controlar sus impulsos y organizando sus experiencias de una manera lógica, y estudia el grado en que las conductas humanas son voluntarias o autodeterminadas, diferenciando diferentes tipos de motivación que varían en función del nivel de autodeterminación. Así, Deci y Ryan (1991) indicaron que la regulación de la conducta podía ser diferenciada en tres grandes bloques: motivación autónoma, controlada y desmotivación.

En primer lugar, la motivación autónoma se divide en regulación intrínseca y regulación identificada, donde la primera de ellas se define como la participación voluntaria en una actividad por el interés, la satisfacción y el placer que se obtiene en el desarrollo de la misma (Bryan \& Solmon, 2007). La regulación identificada está asociada a personas que se involucran en una determinada actividad por la valoración positiva que realizan de la misma, como pueden ser los beneficios relacionados con la salud (Deci \& Ryan, 2000). Del mismo modo, las regulaciones introyectada y externa consti- 
tuyen la motivación controlada (Ryan \& Deci, 2000). La regulación introyectada se corresponde con sujetos que practican una actividad por presión, y por lo tanto, tratan de evitar sentimientos de culpabilidad o vergüenza. La regulación externa aparece cuando una persona participa en una actividad para evitar castigos, obtener premios o para cumplir con las expectativas externas. Por último, la desmotivación constituye el nivel más bajo de autodeterminación, y se caracteriza porque los sujetos no están motivados ni intrínseca ni extrínsecamente y, por lo tanto, no encontrando sentido al por qué seguir practicando la actividad (Deci \& Ryan, 2000).

Esta motivación autodeterminada no aparece de forma espontánea, sino que se necesitan unos antecedentes estables como son el apoyo social y un entorno favorecedor (Deci \& Ryan, 2000). De esta manera, la TAD considera que el comportamiento humano está regulado por tres necesidades psicológicas primarias y universales, que son la necesidad de autonomía, que se caracteriza porque la persona se siente el origen de sus acciones y el guía de sus conductas, la necesidad de competencia, definida como el sentimiento de eficacia de una persona para llevar a cabo una actividad, y la necesidad de relaciones sociales, que hace referencia al sentimiento de pertenencia e integración a un grupo (Deci \& Ryan, 1985). Esta visión tridimensional de las necesidades psicológicas básicas (NPB) es esencial para el desarrollo, tanto de la motivación autodeterminada (Deci \& Ryan, 1985), como para el bienestar psicológico (Ryan \& Deci, 2000). El mayor apoyo y satisfacción de las NPB (Deci \& Ryan, 2008) que ejerza el contexto social (e.g. padres), conducirá a que el individuo alcance una conducta autodeterminada. De esta manera, el apoyo a la autonomía supondrá el empleo de estrategias para atender a los intereses y preferencias de una persona, intentando que tenga el control de sus comportamientos (Deci \& Ryan, 1987) en las acciones deportivas a realizar, el apoyo a la competencia se basa en el uso de estrategias con el objetivo de optimizar la percepción de habilidad de una persona, facilitando el tiempo necesario para alcanzar los objetivos, empleando un feedback positivo y reconociendo el esfuerzo y progreso (Skinner \& Belmont, 1993). Por otro lado, el apoyo a las relaciones sociales hace referencia a los recursos orientados a lograr que una persona se sienta perteneciente y bien relacionado dentro del entorno social que le rodea porque recibe cuidado, afecto y satisfacción (Skinner \& Edge, 2002). En este sentido, Adie, Duda, y Ntoumanis (2012) demostraron que las necesidades de competencia y relaciones sociales predecían en mayor medida una motivación autodeterminada que la autonomía. Asimismo, Balaguer, Castillo, y Duda (2008) encontraron que el apoyo a la autonomía, junto al apoyo a la competencia, predecía la motivación autodeterminada en participantes de diferentes deportes. Así, se ha demostrado que la percepción de apoyo de las tres NPB desemboca en una motivación más autodeterminada (Sánchez-Oliva, Leo, Amado, Campos, \& García-Calvo, 2013; Zhang, Solmon, Kosma, Carson, \& Gu, 2011), conllevando a su vez, a la aparición de consecuencias más positivas como la diversión, persistencia en la actividad, bajos niveles de abandono, menor aburrimiento, etc. (Bois, Sarrazin, Brustad, Trouilloud, \& Cury, 2005; Deci \& Ryan, 1985; Edwardson \& Gorely, 2010; SánchezMiguel et al., 2013; Silva, Matias, Viana, \& Andrade, 2012).

En base a esta relación del entorno social con la práctica de los jóvenes, muchos estudios se han preocupado de analizar la influencia de los padres, demostrando la asociación y prevalencia que existe entre la práctica de actividad que desarrollan ellos y practican sus hijos (Leary, Lilly, Dino, Loprinzi, \& Cottrell, 2013; Loprinzi \& Trost, 2010; Salmon \& Timperio, 2007), destacando los trabajos de Bois, Sarrazin, Brustad, Trouilloud, e Cury (2005), Cheng, Mendonça y Farias Júnior (2014) y Edwarson y Gorely (2010), quiénes demostraron la importancia de los padres en el fomento y realización de actividad física de los hijos/as. 
Sin embargo, son pocos los trabajos que han empleado el análisis de perfiles en padres, y su relación con comportamientos adaptativos en jóvenes. Entre ellos, se destaca el realizado por Swaminathan, Thomas, Yusuf, y Vaz (2013), quiénes encontraron que los padres que pertenecían a un perfil adaptativo (comían más saludable, dormían más y eran más físicamente activos) tenían a hijos que presentaban comportamientos mucho más positivos (tanto a nivel alimenticio, como de hábitos de sueño y, sobre todo de niveles de actividad física). Los autores concluyeron que los hábitos saludables de los jóvenes estaban muy relacionados con el comportamiento de los padres. Otro de los trabajos destacables es el llevado a cabo por Cameron et al. (2011), quiénes hallaron una relación significativa entre el perfil adaptativo de las madres y la mayor cantidad de comportamientos saludables en los hijos (menor cantidad de hábitos sedentarios y mejores hábitos alimenticios).

En cuanto a la influencia de variables psicosociales de los padres sobre los hijos, se destacan los siguientes estudios, aunque en ellos no se desarrollase un análisis de perfiles. SánchezMiguel et al. (2013) hallaron la importancia de la percepción de apoyo de los chicos sobre sus padres en los bajos niveles de desmotivación de los jóvenes. Asimismo, encontraron relaciones significativas entre el apoyo de los padres y la mayor diversión en el contexto de la actividad física y el deporte. Torregrosa et al. (2007) demostraron la asociación entre la percepción de apoyo de los padres con la mayor diversión, compromiso deportivo y menores índices de abandono en la práctica deportiva. Por otro lado, se hallaron relaciones significativas entre la percepción de presión y comportamientos desadaptativos como el abandono, falta de diversión en la práctica y una menor implicación hacia la misma. Sanz-Arazuri, Ponce de León, y Valdemoros (2012), demostraron que el apoyo que los padres daban a las actividades físico - deportivas estaba directamente relacionado con menores posibilidades de que los jóvenes fueran inactivos. Cabe destacar que la mayoría de los trabajos que versan sobre los padres en el contexto de la actividad física y el deporte, no han analizado directamente la influencia de éstos sobre sus hijos/as, sino que han valorado la percepción de los jóvenes deportistas sobre los comportamientos de sus padres (Papaioannou, Ampatzoglou, Kalogiannis, \& Sagovits, 2008).

Teniendo en cuenta todo lo anterior, y tal y como se puede observar, solamente una limitada cantidad de investigaciones han tratado de estudiar la influencia de los padres sobre los jóvenes (Bois et al., 2005). Además, pocos son los trabajos que han analizado la importancia de los padres uniendo constructos de la TAD (apoyo de las tres NPB) y variables de control (presión) y apoyo (implicación) psicológico hacia la práctica deportiva de los hijos. Por el contrario, se destaca que la mayoría de estos trabajos han tratado de analizar variables más biológicas que psicosociales. De esta manera, la generación de perfiles nos proporciona una perspectiva aplicada en el trabajo, ya que nos puede instruir acerca del porcentaje de padres que pueden presentar unas determinadas características en el contexto deportivo (Breckenridge, 2000). Esta información podría ser útil tanto desde el punto de vista del conocimiento de las causas que implican a los padres en la práctica deportiva, como desde la perspectiva de intervención en dicho contexto.

Por todo ello, el principal objetivo de este estudio era analizar los perfiles de padres en cuanto a su percepción de presión, implicación y apoyo a las NPB en el deporte, e investigar el tipo de relación de cada uno de estos perfiles con la motivación y el aburrimiento desarrollado por sus hijos. Asimismo, se pretende analizar estos perfiles y su relación con los niveles de práctica deportiva de los padres. De acuerdo con los objetivos formulados en la investigación, las hipótesis que se plantearon fueron las siguientes. En primer lugar, se plantea como hipótesis la creación de un perfil con elevada percepción de presión de los padres y bajos niveles de apoyo a las NPB y percepción de implicación, y otro perfil con bajos niveles de 
percepción de presión y alta percepción de apoyo de las NPB e implicación. Asimismo, la segunda hipótesis enuncia que un perfil positivo de los padres en variables adaptativas como el apoyo a las NPB e implicación supondría altos niveles de práctica deportiva de éstos. Finalmente, la tercera hipótesis formulada indica que un perfil adaptativo en los padres estaría relacionado con una motivación autónoma en los jóvenes deportistas, mientras que un perfil desadaptativo se asociaría con la motivación controlada y la desmotivación en los jóvenes deportistas.

\section{MÉTODO}

\section{Muestra}

La muestra del estudio estuvo formada por 301 padres de jóvenes participantes federados en deporte escolar, tanto de deportes individuales $(n=128)$ como colectivos $(n=191)$, con edades comprendidas entre los 11 y los 16 años $(M=13.92 ; \mathrm{DT}=2.04)$. La selección de los participantes fue realizada por muestreo aleatorio por conglomerados, formado por algunos clubes adscritos a los Judex (Juegos Deportivos Extremeños en Edad Escolar). En relación a las normas de cualquier investigación científica, el estudio previamente recibió la aprobación del Comité de Ética de la Universidad de Extremadura. Los padres y deportistas fueron tratados de acuerdo con las directrices de la American Psychological Association de acuerdo al consentimiento, anonimato y privacidad.

\section{Instrumentos}

Percepción de los padres de apoyo a las Necesidades Psicológicas Básicas

Para valorar la percepción de los padres del apoyo a las Necesidades Psicológicas Básicas, se utilizó una adaptación al contexto deportivo del Cuestionario de Apoyo a las Necesidades Psicológicas Básicas (CANPB: Sánchez-Oliva et al., 2013). Esta escala está compuesta por la frase introductoria «En el deporte de mi hijo/a...», seguida de 12 ítems (cuatro por cada factor), que valoran el apoyo a la autonomía (4 ítems, ej.: "Pregunto a menudo sus preferencias con respecto al deporte a realizar"), el apoyo a la competencia (4 ítems, ej.: "Animo a que confíe en sus capacidades para hacer bien las tareas), y el apoyo a las relaciones sociales (4 ítems, ej.: "Favorezco el buen ambiente entre todos los compañeros"). Como el instrumento utilizado fue adaptado para el desarrollo de esta investigación y aún no ha sido validado en el ámbito deportivo, se llevó a cabo un Análisis Factorial Confirmatorio (AFC), en el que se obtuvieron los siguientes índices de ajuste: $\chi^{2} / \mathrm{df}=5.12$; CFI $=.96$; $\mathrm{TLI}=.95$; GFI $=.96 ; \mathrm{SRMR}=.04$; $\mathrm{y}$ RMSEA $=.06$; comprobándose que se ajustaba correctamente a la población objeto de estudio. En relación a la consistencia interna del cuestionario, ésta resultó ser adecuada, ya que el apoyo a la autonomía obtuvo un valor .73, el apoyo a la competencia mostró un valor de .81 , mientras que el apoyo a las relaciones sociales obtuvo un valor de .85 .

Participación de los padres en la práctica deportiva

Para valorar la participación de los padres en la práctica deportiva se utilizó la versión validada al castellano por García-Calvo, Leo, Sánchez-Oliva, Amado y Sánchez-Miguel (2011) del Parental Involvement Sport Questionaire (PISQ: Wuerth, Lee, \& Alfermann, 2004). El cuestionario está formado por un total de 16 ítems, que se agrupan en cuatro factores. Se emplearon 8 de los 16 ítems para valorar la percepción sobre la implicación activa (4 ítems, ej.: "Ayudo voluntariamente a la organización de las competiciones, ayudo al entrenador, anoto en las competiciones, etc.") y percepción de la presión que le ejercen a sus hijos (4 ítems, ej.: "Le meto presión para que gane”). El análisis de consistencia interna arrojó valores adecuados de .79 para la percepción de la presión y .82 para la percepción de implicación. 


\section{Motivación de los deportistas.}

Para valorar la motivación de los deportistas se utilizó una adaptación al deporte del Cuestionario de Motivación en la Educación Física (CMEF: Sánchez-Oliva, Leo, Amado, GonzálezPonce, \& García-Calvo, 2012). Esta escala empieza con la frase introductoria "Yo participo en el deporte...", seguida de 20 ítems (4 por factor) que miden la motivación intrínseca (4 ítems, ej.: "Porque el deporte es divertido"), regulación identificada (4 ítems, ej.: "Porque este deporte me aporta conocimientos y habilidades que considero importantes"), regulación introyectada (4 ítems, ej.: "Porque lo veo necesario para sentirme bien conmigo mismo"), regulación externa (4 ítems, ej.: "Para demostrar al entrenador/a y compañeros/as mi interés por el deporte") y desmotivación (4 ítems, ej.: "Pero realmente siento que estoy perdiendo mi tiempo con este deporte"). Como el instrumento de medida aún no ha sido validado en el deporte, se llevó a cabo un análisis factorial confirmatorio, que obtuvo los siguientes índices de ajuste: $\chi^{2} / \mathrm{df}=4.80$; CFI $=.92$; TLI $=.91 ; \mathrm{GFI}=.93 ;$ SRMR $=.05 ;$ y RMSEA $=$ .06; demostrando una correcta adecuación. Los índices de fiabilidad mostraron puntuaciones apropiadas de .72 para la motivación intrínseca, .75 para la regulación identificada, .77 para la regulación introyectada, .80 para la regulación externa y .77 para la desmotivación.

\section{Aburrimiento.}

Se utilizó una versión adaptada de la Escala Disfrute/Aburrimiento en el deporte (Duda \& Nicholls, 1992), validada en el contexto español por Baena-Extremera, Granero-Gallegos, Bracho-Amador, y Pérez-Quero (2012). La frase original referida a la educación física fue modificada (e.g., "En Educación Física, yo normalmente espero que las clases finalicen rápidamente" por "Cuando practico deporte, yo normalmente espero que los entrenamientos finalicen rápidamente"). El instrumento original consta de 8 ítems: 4 ítems que miden diversión y 4 ítems que valoran aburrimiento. Se eligieron los 4 ítems que componían el fac- tor aburrimiento, y de esos, finalmente se decidió incluir solamente 3 ítems en el factor, ya que uno de ellos no mostró un adecuado peso factorial (>.30). Finalmente, se indica que el índice de fiabilidad de este factor fue de .85 .

Las respuestas a los cuestionarios anteriormente descritos, estaban valoradas en una escala tipo Likert con un rango de respuesta de 1 a 5 , en la que el 1 correspondía a totalmente en desacuerdo y el 5 a totalmente de acuerdo con la formulación de la frase.

\section{Procedimientos}

Antes de llevar a cabo la investigación se informó a todos los implicados sobre el proceso que se iba a seguir, incidiendo en que la participación era voluntaria y que los datos serían tratados de forma confidencial, obteniendo un consentimiento informado de los entrenadores de los clubs y de los padres de los deportistas.

Los participantes rellenaron el cuestionario mediante la aplicación Google Docs (Deportistas: http://goo.gl/Sli5i; Padres: http://goo.gl/G3ejJ). Anteriormente a esto, el investigador principal se reunió con los entrenadores y padres de los jóvenes deportistas, con el objetivo de explicar los instrumentos empleados y resolver cualquier duda que pudiera surgir. El tiempo empleado para rellenar el cuestionario fue de 20 minutos aproximadamente.

\section{Análise estatística}

Inicialmente, se calcularon los estadísticos descriptivos y el coeficiente de correlación entre las distintas variables incluidas en el estudio. A continuación, se llevó a cabo un análisis de conglomerados (Breckenridge, 2000), con el objetivo de encontrar grupos de padres con perfiles similares. Más tarde, se desarrolló la prueba de Chi-Cuadrado de Pearson, junto con un análisis de residuos, con el fin de analizar la distribución de los perfiles encontrados en función de la práctica deportiva semanal. Por último, para comprobar si existían diferencias en la motivación de los deportistas en función 
de los perfiles creados en los padres, se llevó a cabo un análisis de varianza (ANOVA).

\section{RESULTADOS}

\section{Estadísticos descriptivos y análisis de corre-} lación

En la tabla 1, se muestran los resultados del análisis descriptivo y de correlaciones bivariadas utilizando el coeficiente de correlación de Pearson. Respecto a los resultados descriptivos, se observó cómo los valores de apoyo a las relaciones sociales, competencia y autonomía presentaron las puntuaciones más altas, segui- das por la implicación de los padres, en contraposición con la presión de los padres.

Por otro lado, el análisis de correlación mostró relación positiva y significativa entre la percepción de apoyo a las NPB, la percepción de implicación de los padres y la motivación autónoma de los hijos. Sin embargo, se pudo observar una significatividad negativa y muy alta entre el apoyo a las NPB y la implicación de los padres, con la motivación controlada y la desmotivación, mientras que la percepción de presión tenía una correlación positiva con la motivación controlada y desmotivación.

Tabla 1.

Media, desviación típica y correlaciones entre las variables del estudio

\begin{tabular}{|c|c|c|c|c|c|c|c|}
\hline Variables & $M$ & $D T$ & 1 & 2 & 3 & 4 & 5 \\
\hline 1. Apoyo Autonomía Padres & 4.43 & 0.63 & - & & & & \\
\hline 2. Apoyo Competencia Padres & 4.54 & 0.62 & $.734^{* *}$ & - & & & \\
\hline 3. Apoyo Relaciones Sociales Padres & 4.72 & 0.53 & $.686^{* *}$ & $.803^{* *}$ & - & & \\
\hline 4. Presión Padres & 1.93 & 0.84 & $-.195^{* *}$ & $-.173 * *$ & $-.231^{* *}$ & - & \\
\hline 5. Implicación Padres & 4.18 & 0.62 & $.484^{* *}$ & $.589^{* *}$ & $.509 * *$ & -.097 & - \\
\hline
\end{tabular}

${ }^{*} p<.05 ;{ }^{* *} p<.01$

\section{Análisis de conglomerados para la obtención de los perfiles}

Para conocer los perfiles que podrían surgir de la muestra, se incluyeron el apoyo a las NPB, la implicación y presión de los padres, y posteriormente se realizó un análisis de conglomerados combinando métodos jerárquicos y no jerárquicos (Hair, Anderson, Tatham, \& Black, 1998). En primer lugar, con el objetivo de reducir el impacto de los valores atípicos, se eliminaron los valores superiores a tres veces la desviación típica por encima o por debajo de la media en cualquiera de las variables incluidas en el análisis. Posteriormente, se realizó un análisis de conglomerados jerárquico mediante el modelo de Ward, estandarizando las variables y utilizando las puntuaciones Z. En la tabla 2, se pueden observar las medias, desviaciones típicas y puntuaciones $\mathrm{Z}$ de las variables del estudio en cada perfil. Las puntuaciones $\mathrm{Z}$ de \pm 0.5 o mayores se emplearon como criterio para describir si un grupo puntuaba relativamente alto o bajo comparado con el resto (Wang \& Biddle, 2001). Tras la realización del análisis, el dendograma sugirió dos conglomerados como la solución más conveniente. No obstante, debido al carácter exploratorio del análisis jerárquico, era recomendable confirmar los resultados obtenidos a través de otro tipo de técnica no jerárquica. Para ello, se llevó a cabo un análisis de conglomerados K-medias, que nos informó del grado de similitud existente entre el número de conglomerados encontrados en los análisis jerárquicos y no jerárquicos. Los resultados de los dos análisis fueron muy consistentes, tanto en la magnitud, como en los patrones de los perfiles, apoyando la estabilidad de los dos perfiles.

Como se puede apreciar en la figura 1, el primer perfil está compuesto por 82 padres y se caracteriza por tener valores bajos de apoyo a las NPB y la implicación, contrastando con una elevada presión. El segundo conglomerado está formado por 239 padres y se define por tener puntuaciones altas en la percepción de apoyo a las NPB y la implicación hacia la práctica deportiva de sus hijos/as, contrastando con bajos valores de presión. 
Tabla 2.

Medias, desviaciones típicas y puntuaciones $Z$ de las variables en cada cluster

\begin{tabular}{rlcccccc}
\hline & & \multicolumn{3}{c}{$\begin{array}{c}\text { Cluster 1 } \\
(n=82)\end{array}$} & \multicolumn{4}{c}{$\begin{array}{c}\text { Cluster 2 } \\
(n=239)\end{array}$} \\
\hline Variables & & $M$ & $D T$ & $Z$ & $M$ & $D T$ & $Z$ \\
\hline 1. & Apoyo Autonomía Padres & 3.70 & 0.61 & -5.43 & 4.68 & 0.39 & 0.89 \\
2. & Apoyo Competencia Padres & 3.71 & 0.63 & -5.65 & 4.83 & 0.26 & 0.72 \\
3. & Apoyo Relaciones Sociales Padres & 4.09 & 0.71 & -6.91 & 4.94 & 0.18 & 0.52 \\
4. & Presión Padres & 2.19 & 0.87 & 3.64 & 1.85 & 0.81 & -1.11 \\
5. & Implicación Padres & 3.59 & 0.58 & -4.03 & 4.38 & 0.49 & 1.31 \\
\hline
\end{tabular}

Como se puede apreciar en la figura 1 , el primer perfil está compuesto por 82 padres y se caracteriza por tener valores bajos de apoyo a las NPB y la implicación, contrastando con una elevada presión. El segundo conglomerado está formado por 239 padres y se define por tener puntuaciones altas en la percepción de apoyo a las NPB y la implicación hacia la práctica deportiva de sus hijos/as, contrastando con bajos valores de presión.

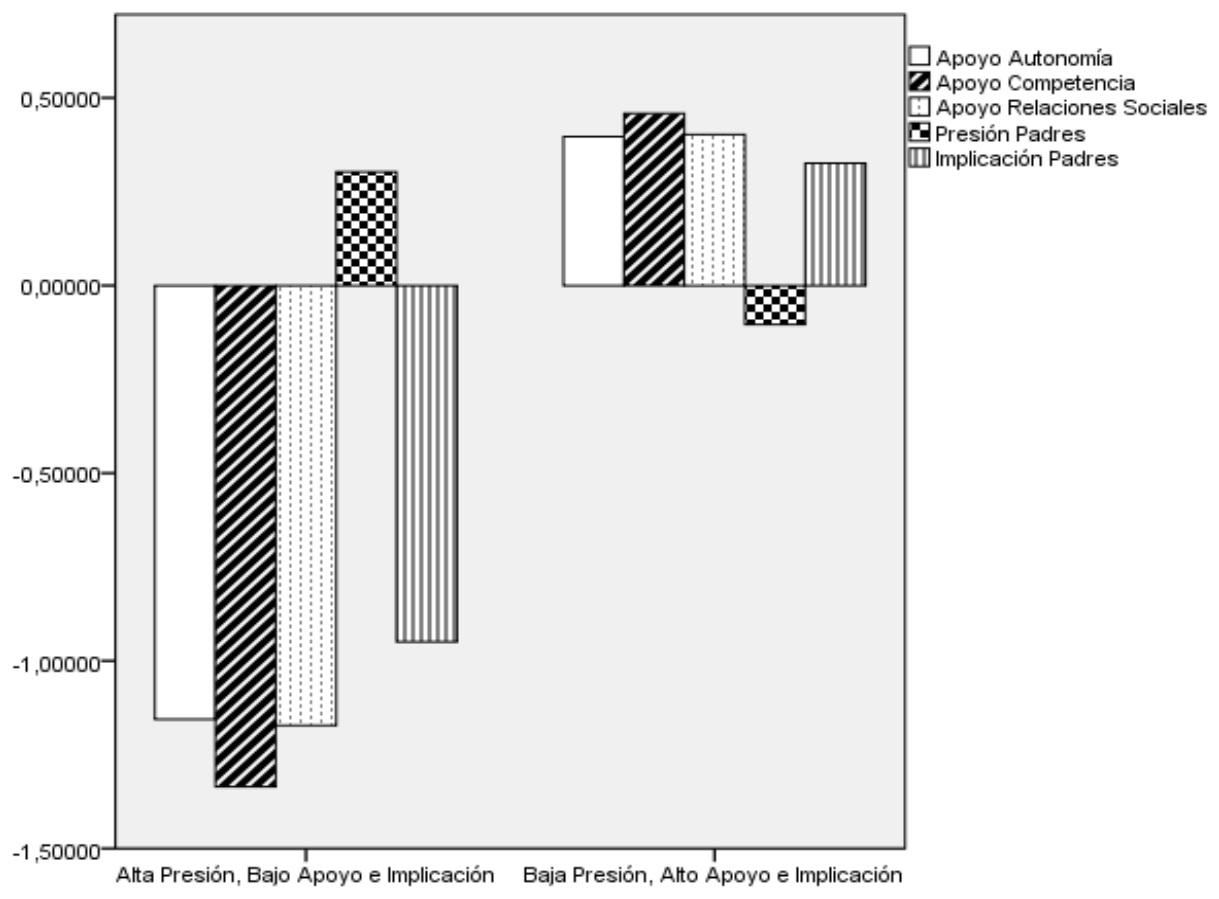

Figura 1. Perfiles motivacionales obtenidos a partir del análisis de conglomerados.

\section{Análisis de las diferencias}

Con el objetivo de comprobar las diferencias en la distribución de los perfiles encontrados en función de la actividad física realizada por los padres, se decidió realizar un análisis de diferencias a través del estadístico Chi Cuadrado de Pearson. Esta prueba de bondad de ajuste, compara las frecuencias observadas y esperadas en cada categoría, para contrastar si todas las categorías contienen la misma proporción de valores o si cada categoría contiene una proporción de valores especificada por el usuario. Un valor en el nivel de significación observado $(p<.05)$, informa que existen diferencias significativas entre las frecuencias esperadas y las observadas. Asimismo, la utilización de los residuos tipificados corregidos, nos aporta información sobre donde se encuen- 
tran estas diferencias, ya que los residuos iguales o mayores de 1.90 , son considerados como indicadores de que existe dependencia entre esas dos categorías y que por tanto las diferencias son significativas.

En la tabla 3 se puede apreciar, al analizar las frecuencias y los residuos, que en el perfil relacionado con las variables de alta presión, bajo apoyo e implicación, no aparecían diferencias significativas en los padres que no realizan ninguna actividad, donde el recuento fue superior a la frecuencia esperada, y en 1-3 horas semanales, donde el recuento fue inferior a la frecuencia esperada. Sin embargo, los padres con perfil caracterizado por baja presión, alto apoyo e implicación presentaron un recuento menor al esperado en la categoría de ningún tipo de actividad física, y un recuento mayor en la práctica de 1-3 horas semanales.

Para examinar el nivel de significación de estos datos, se utilizó el estadístico Chi Cuadrado de Pearson cuyo valor fue $\chi 2=15.51$ con una $\mathrm{p}<.001$, lo que corrobora la existencia de diferencias significativas en función de las horas de práctica de actividad física semanales.

Tabla 3.

Tabla de contingencia de los perfiles motivacionales en función de la actividad física realizada por los padre

\begin{tabular}{|c|c|c|c|c|c|c|}
\hline Perfiles & Valores & $\begin{array}{l}\text { Ninguna } \\
\text { práctica }\end{array}$ & $\begin{array}{l}\text { 1-3 horas } \\
\text { semanales }\end{array}$ & $\begin{array}{l}\text { 4-6 horas } \\
\text { semanales }\end{array}$ & $\begin{array}{c}\text { Más de } 6 \text { horas } \\
\text { semanales }\end{array}$ & Total \\
\hline \multirow{3}{*}{$\begin{array}{l}\text { Alta Presión, } \\
\text { Bajos Apoyos }\end{array}$} & Recuento & 47 & 17 & 14 & 4 & 82 \\
\hline & Frecuencia esperada & 32.2 & 25.3 & 17.4 & 7.2 & 82 \\
\hline & Residuos corregidos & 3.9 & -2.3 & -1.1 & -1.4 & \\
\hline \multirow{3}{*}{$\begin{array}{l}\text { Baja Presión, } \\
\text { Altos Apoyos }\end{array}$} & Recuento & 79 & 82 & 54 & 24 & 239 \\
\hline & Frecuencia esperada & 93.8 & 73.7 & 50.6 & 20.8 & 239 \\
\hline & Residuos corregidos & -3.9 & 2.3 & 1.1 & 1.4 & \\
\hline
\end{tabular}

Nota. Los valores de los residuos corregidos superiores a 1.9 son considerados significativos.

Posteriormente, con el objetivo de comprobar las diferencias existentes en la distribución de los perfiles encontrados en función de la variables motivacionales en los deportistas, así como en el aburrimiento de éstos, se decidió realizar un análisis de diferencias (ANOVA).
En la tabla 4, se puede observar cómo el perfil más desadaptativo obtuvo puntuaciones significativamente más bajas en la motivación autónoma de los adolescentes, y más altas en la desmotivación de los deportistas y en el aburrimiento de éstos en la práctica deportiva.

Tabla 4.

Análisis de diferencias de los perfiles en función de las variables estudiadas

\begin{tabular}{lccccc}
\hline & $\begin{array}{c}\text { Alta Presión/ Bajo Apoyo e } \\
\text { Implicación }\end{array}$ & $\begin{array}{c}\text { Baja Presión/Alto Apoyo e } \\
\text { Implicación }\end{array}$ & MC & $F$ & $p$ \\
\hline 1. Motivación Autónoma & $4.36 \pm 0.52$ & $4.49 \pm 0.52$ & 1.06 & 3.96 & 0.047 \\
2. Motivación Controlada & $2.98 \pm 1.00$ & $3.01 \pm 1.01$ & .11 & 1.08 & 0.743 \\
3. Desmotivación & $1.45 \pm 0.89$ & $1.27 \pm 0.60$ & 1.93 & 4.09 & 0.044 \\
4. Aburrimiento & $1.45 \pm 0.95$ & $1.26 \pm 0.58$ & 2.13 & 4.43 & 0.036 \\
\hline
\end{tabular}

\section{DISCUSIÓN}

El principal objetivo de este estudio era analizar los perfiles existentes en los padres en cuanto a su percepción de presión, implicación y apoyo a las NPB en el deporte, e investigar el tipo de relación de cada uno de estos perfiles con la motivación y el aburrimiento desarrollado por sus hijos. Asimismo, se pretendió analizar estos perfiles y su relación con los niveles de práctica deportiva de los padres. En relación con los objetivos formulados en la investigación, se establecieron diferentes hipótesis. 
En primer lugar, se planteó como hipótesis la creación de un perfil con elevada percepción de presión de los padres y bajos niveles de apoyo a las NPB y percepción de implicación, y otro perfil con bajos niveles de percepción de presión y alta percepción de apoyo de las NPB e implicación. Si se observan los resultados del análisis de conglomerados, se confirma la existencia de dos perfiles de padres, revelando patrones diferentes en los procesos regulatorios respecto a la actividad física y deportiva de sus hijos/as. El primero de ellos denominado perfil presión, donde se apreciaba alta percepción de presión, baja implicación y bajo apoyo a las NPB, y otro perfil denominado perfil apoyo donde se observaba baja percepción de presión, alta implicación y apoyo a las NPB. Si se observan las características de los perfiles, se puede apreciar cómo se han agrupado correctamente y atendiendo a dos tipos de padres que se pueden encontrar en el contexto de la actividad física y el deporte, los padres que apoyan y los padres que presionan a sus hijos en la actividad física y deportiva (SánchezMiguel et al., 2013; Wuerth et al., 2004).

Estos resultados tienen importantes implicaciones teóricas, además de aumentar el conocimiento sobre la incidencia de los padres en el contexto de la actividad física y el deporte de sus hijos. Como se muestran en los resultados, los padres que percibían una alta presión hacia sus hijos, tienen un bajo apoyo a las NPB e implicación hacia la actividad. De forma contraria, los padres que percibían una implicación y apoyo hacia las NPB, son los que percibían que ejercían menor presión sobre sus hijos. Se destaca que solamente el $27,24 \%$ de los padres analizados se encuadran en el perfil de presión, mientras que el $72,76 \%$ de los padres se incluían en el perfil apoyo. Estos resultados son consistentes con otros hallados por SánchezMiguel et al. (2013) y Torregrosa et al. (2007) quiénes encontraron mayor número de frecuencia de padres que se implicaban y apoyaban la práctica deportiva de sus hijos.

Con respecto a la segunda hipótesis que enunciaba que un perfil positivo de los padres en variables adaptativas como el apoyo a las NPB e implicación supondría altos niveles de práctica deportiva de éstos. Esta hipótesis se confirma tras observar los resultados de la tabla de contingencia. Así, se destaca que los padres con perfiles de apoyo son los que realizan mayor cantidad de actividad física durante la semana, en comparación con aquellos que presentan un perfil presión. Es importante enfatizar este resultado, ya que se ha demostrado que el nivel de actividad física de los padres está directamente relacionado con la cantidad de práctica deportiva realizada por los hijos (Cheng et al., 2014). Además de ello, este resultado se refuerza debido a que los padres activos implican hijos activos dado que conductualmente no pueden hacer otra cosa, hasta que se hallan en condiciones de tomar decisiones autónomas. Estos hallazgos han sido apoyados por muchos trabajos, indicando que en general, padres activos es más probable que tengan hijos activos (Edwardson \& Gorely, 2010; Seabra, Mendonça, Thomis, Anjos, \& Maia, 2008). Edwardson y Gorely (2010) en su revisión sistemática hallaron la enorme importancia que tienen los padres en el fomento de la actividad física (tanto moderada y vigorosa, como aquella que se puede realizar en tiempo libre) en los niños de 6 a 11 años, gracias a que su realización de actividad es un modelo a seguir, y se implican mucho más en la actividad física y deportiva de sus hijos. Asimismo, los autores hallaron que las actitudes de los padres hacia la actividad física, su nivel de práctica y la implicación en la misma, eran variables importantes para que sus hijos/as adolescentes (12 18 años) se mantuvieran físicamente activos. Por todo ello, si se consiguiera aumentar la cantidad de práctica de actividad deportiva por parte de los padres, seguramente que éstos tendrían un comportamiento de implicación y apoyo a las NPB de los hijos, además de fomentar en mayor medida la práctica deportiva de éstos.

Finalmente, la tercera hipótesis formulada indica que un perfil adaptativo en los padres estaría relacionado con una motivación autó- 
noma en los jóvenes deportistas, mientras que un perfil desadaptativo se asociaría con una motivación controlada y la desmotivación en los jóvenes deportistas. En relación a esta hipótesis, Deci y Ryan (1985) indicaron en su explicación sobre la TAD, que el apoyo a las NPB y el tipo de regulación motivacional permiten explicar determinadas consecuencias adaptativas (a nivel cognitivo, social, comportamental, afectivo, etc). En este sentido, los resultados sugieren cómo los padres son responsables en la modelación de comportamientos como el interés o aburrimiento hacia la práctica deportiva. Este apoyo social hacia la práctica deportiva ya ha sido demostrado por otros trabajos, quienes encontraron a los padres como determinantes importantes en la realización de actividad física por parte de los hijos (Cheng et al., 2014). Otros trabajos como el de Bois et al. (2005) demostraron la importancia que tienen los padres (tanto el padre como la madre) en el apoyo a la competencia de los jóvenes y, sus consecuencias como la mayor implicación en la práctica de actividad física. Asimismo, Edwarson y Gorely (2010) concluyeron que el comportamiento que los padres mantienen en la práctica deportiva de sus hijos, es una variable importante para que sus hijos tengan más consecuencias positivas como el mantenerse físicamente activos. Por otro lado, Wilson y Spink (2010) demostraron que el mayor apoyo social por parte de los padres conducía a un mejor y mayor cambio de conducta hacia la actividad física y deportiva de los hijos. En esta línea, Davidson y Jago (2009) hallaron que comparando con chicas entre 9 y 15 años que no mantuvieron unos niveles de actividad físicodeportiva medios, las chicas que presentaron niveles medio - altos de práctica, tenían padres que servían como modelos en cuanto a la realización de práctica físico deportiva. Asimismo, se destaca que estos padres mantenían un alto apoyo hacia la actividad de sus hijas.

El hallazgo de que los jóvenes cuyos padres se encuentran en el perfil presión perciben un mayor aburrimiento en la práctica deportiva, se puede explicar mediante los postulados de
Russell (1987), quién demostró que la percepción de aburrimiento de los jóvenes es debido entre otros factores a una baja percepción de apoyo social, principalmente de sus padres. De esta manera, nuestros resultados siguen este postulado, ya que los padres del perfil presión (aquellos que apoyan menos a sus hijos), tienen hijos con una mayor percepción de aburrimiento en la práctica.

Por otro lado, se muestra cómo el perfil presión está relacionado con bajos niveles de motivación en los jóvenes deportistas, apreciándose diferencias significativas en cuanto a la desmotivación de los hijos entre los dos perfiles de padres. De esta manera, un comportamiento de presión por parte de los padres, unido a un bajo apoyo de las NPB conducen, entre otras consecuencias, a que los jóvenes tengan un mayor desinterés hacia la práctica deportiva. Estos resultados ya han sido encontrados previamente por Sánchez-Miguel et al. (2013) quiénes hallaron que los jóvenes deportistas que percibían mayor presión por parte de los padres, presentaban también mayores niveles de desmotivación en la práctica de actividad deportiva.

\section{CONCLUSIONES}

Para concluir, se puede destacar que comportamientos de presión y bajo apoyo de las NPB en los padres, puede conllevar a una desmotivación en la práctica deportiva de los hijos, y un mayor aburrimiento de éstos. Por otro lado, se destaca que los padres con perfiles adaptativos son aquellos que realizan significativamente una mayor cantidad de práctica deportiva durante la semana, en comparación con aquellos padres que tienen un perfil más desadaptativo. Es importante destacar que este estudio mide directamente la percepción de los padres hacia el apoyo y/o presión hacia la práctica deportiva de sus hijos. La mayoría de estudios previos han estudiado la percepción de los hijos sobre sus padres. Asimismo, se destaca que, hasta nuestro conocimiento, no se han realizado ningún trabajo de perfiles con padres 
en el contexto de la actividad física y deportiva bajo la perspectiva teórica de la TAD.

Asimismo, y teniendo en cuenta los resultados obtenidos en el estudio, se muestran algunas implicaciones prácticas para desarrollar en el contexto de la actividad deportiva escolar. Por un lado, informar a los clubes deportivos de la importancia que adquieren no solamente los entrenadores en el proceso formativo-deportivo, sino también los padres en la generación de mejores consecuencias a nivel comportamental. En este sentido, se recomienda realizar programas de formación continuos a todos los implicados en la práctica. Por otro lado, los resultados nos sugiere la promoción de la actividad física y deportiva en gente adulta, mediante programas específicos, mejoras estructurales en las ciudades, información sobre la importancia de hábitos de vida, etc., que de una manera indirecta repercuta en la mejor adquisición de hábitos saludables en los jóvenes deportistas en edad escolar.

En relación a las limitaciones del estudio, se destaca la incapacidad de establecer relaciones causales entre las variables, ya que el estudio es descriptivo y como señalan algunos trabajos (Edwardson \& Gorely, 2010; Sánchez-Miguel et al., 2013) son varios los significativos que pueden incidir en la práctica deportiva de los hijos. Igualmente, otra limitación del trabajo es que se ha evaluado la percepción de los padres en su conjunto, sin diferenciar entre padres y madres, lo que nos facilitaría unos datos más aproximados de la realidad en cuanto a la incidencia en la realización de actividad deportiva de los hijos.

Como prospectivas, se indica la posibilidad de realizar estudios longitudinales, con el objetivo de conocer de manera más profunda los procesos motivacionales relacionados con el apoyo social, que determinan la mayor o menor cantidad de práctica de actividad deportiva en los jóvenes. Asimismo, se destaca la necesidad de analizar directamente la conducta de los padres en la práctica deportiva (tipos de comunicación, conductas desarrolladas durante la competición...), para conocer la relación con las consecuencias desarrolladas por los hijos. Por otro lado, otra futura línea de investigación importante a desarrollar, sería analizar exactamente la cantidad de práctica que realizan los jóvenes participantes, y relacionarlo con los tipos de motivación de los otros significativos.

\section{Agradecimientos:}

Nada a declarar

\section{Conflicto de intereses:}

Nada a declarar.

Financiación:

Consejo Superior de Deportes

\section{REFERENCIAS}

Adie, J. W., Duda, J. L., \& Ntoumanis, N. (2012). Perceived coach-autonomy support, basic need satisfaction and the well- and ill-being of elite youth soccer players: A longitudinal investigation. Psychology of Sport and Exercise, 13(1), 51-59.

http://doi.org/10.1016/j.psychsport.2011.07.0 08

Baena-Extremera, A., Granero-Gallegos, A., BrachoAmador, C., \& Pérez-Quero, F. J. (2012). Spanish Version of the Sport Satisfaction Instrument (SSI) Adapted to Physical Education. Journal of Psychodidactics, 17(2), 377-395. http://doi.org/10.1387/RevPsicodidact.4037

Balaguer, I., Castillo, I., \& Duda, J. L. (2008). Apoyo a la autonomía, satisfacción de las necesidades, motivación y bienestar en deportistas de competición: un análisis de la teoría de la autodeterminación. Revista de Psicología del Deporte, 17(1), 123-139.

Bois, J. E., Sarrazin, P. G., Brustad, R. J., Trouilloud, D. O., \& Cury, F. (2005). Elementary schoolchildren's perceived competence and physical activity involvement: the influence of parents' role modelling behaviours and perceptions of their child's competence. Psychology of Sport and Exercise, 6(4), 381-397. http://doi.org/10.1016/j.psychsport.2004.03.0 03

Breckenridge, J. N. (2000). Validating Cluster Analysis: Consistent Replication and Symmetry. Multivariate Behavioral Research, 35(2), 261- 
285.

http://doi.org/10.1207/S15327906MBR3502_ 5

Bryan, C. L., \& Solmon, M. A. (2007). SelfDetermination in physical education: Designing class environments to promote active lifestyles current status of children's physical activity. Journal of Teaching in Physical Education, 26(3), 260-278.

Cameron, A. J., Crawford, D. A., Salmon, J., Campbell, K., McNaughton, S. A., Mishra, G. D., \& Ball, K. (2011). Clustering of obesity-related risk behaviors in children and their mothers. Annals of Epidemiology, 21(2), 95-102. http://doi.org/10.1016/j.annepidem.2010.11.0 01

Cheng, L. A., Mendonça, G., \& Farias Júnior, J. C. de. (2014). Physical activity in adolescents: analysis of the social influence of parents and friends. Jornal De Pediatria, 90(1), 35-41. http://doi.org/10.1016/j.jped.2013.05.006

Davison, K. K., \& Jago, R. (2009). Change in parent and peer support across ages 9 to $15 \mathrm{yr}$ and adolescent girls' physical activity. Medicine and Science in Sports and Exercise, 41(9), 18161825.

http://doi.org/10.1249/MSS.0b013e3181a278 e2

Deci, E. L., \& Ryan, R. M. (1985). Intrinsic Motivation and Self-Determination in Human Behavior. New York: Plenum.

Deci, E. L., \& Ryan, R. M. (1987). The support of autonomy and the control of behavior. Journal of Personality and Social Psychology, 53(6), 1024-1037.

Deci, E. L., \& Ryan, R. M. (1991). A motivational approach to self: integration in personality. Em R. Dienstbier (Ed.), Nebraska Symposium on Motivation: Perspectives on Motivation (pp. 237-288). Lincoln: University of Nebraska Press.

Deci, E. L., \& Ryan, R. M. (2000). The «What» and "Why" of Goal Pursuits: Human Needs and the Self-Determination of Behavior. Psychological Inquiry, 11(4), 227-268. http://doi.org/10.1207/S15327965PLI1104_01

Deci, E. L., \& Ryan, R. M. (2008). SelfDetermination Theory: A Macrotheory of Human Motivation, Development, and Health. Canadian Psychology, 49(3), 182-185. http://doi.org/10.1037/a0012801

Duda, J. L., \& Nicholls, J. G. (1992). Dimensions of achievement motivation in schoolwork and sport. Journal of Educational Psychology, 84(3), 290-299. http://doi.org/10.1037/00220663.84.3.290

Edwardson, C. L., \& Gorely, T. (2010). Parental influences on different types and intensities of physical activity in youth: A systematic review. Psychology of Sport and Exercise, 11(6), 522-
535.

http://doi.org/10.1016/j.psychsport.2010.05.0 01

García-Calvo, T., Leo, F. M., Sánchez-Oliva, D., Amado, D., \& Sánchez-Miguel, P. A. (2011). Validation of the Parental Involvement in Sport Questionnaire in Spanish Population. Apresentado na XIII Congreso de Psicología del Deporte, Madeira.

Hair, J. F., Anderson, R. E., Tatham, R. L., \& Black, W. C. (1998). Multivariate Data Analysis (5a ed.). New Jersey: Prentice Hall. Obtido de http://www.abebooks.com/Multivariate-DataAnalysis-Hair-JosephAnderson/6927003336/bd

Holt, N. L., Tamminen, K. A., Black, D. E., Mandigo, J. L., \& Fox, K. R. (2009). Youth sport parenting styles and practices. Journal of Sport \& EXercise Psychology, 31(1), 37-59.

Leary, J. M., Lilly, C. L., Dino, G., Loprinzi, P. D., \& Cottrell, L. (2013). Parental influences on 7-9 year olds' physical activity: a conceptual model. Preventive Medicine, 56(5), 341-344. http://doi.org/10.1016/j.ypmed.2013.02.005

Loprinzi, P. D., \& Trost, S. G. (2010). Parental influences on physical activity behavior in preschool children. Preventive Medicine, 50(3), 129-133. http://doi.org/10.1016/j.ypmed.2009.11.010

Papaioannou, A. G., Ampatzoglou, G., Kalogiannis, P., \& Sagovits, A. (2008). Social agents, achievement goals, satisfaction and academic achievement in youth sport. Psychology of Sport and Exercise, 9(2), 122-141. http://doi.org/10.1016/j.psychsport.2007.02.0 03

Russel, R. V. (1987). The relative contribution of recreation satisfaction and activity participation to the life satisfaction of retirees. Journal of Leisure Research, 19, 329-340.

Ryan, R. M., \& Deci, E. L. (2000). Selfdetermination theory and the facilitation of intrinsic motivation, social development, and well-being. The American Psychologist, 55(1), 68-78.

Salmon, J., \& Timperio, A. (2007). Prevalence, trends and environmental influences on child and youth physical activity. Medicine and Sport Science, $\quad 50, \quad 183-199$. http://doi.org/10.1159/0000101391

Sánchez-Miguel, P. A. (2010). Estudio del abandono deportivo en escolares extremeños (Doutoramento). Universidad de Extremadura, Badajoz. Obtido de http://dialnet.unirioja.es/servlet/tesis?codigo $=22550$

Sánchez-Miguel, P. A., Leo, F. M., Sánchez-Oliva, D., Amado, D., \& García-Calvo, T. (2013). The Importance of Parents' Behavior in their Children's Enjoyment and Amotivation in Sports. 
142 | PA Sánchez-Miguel, JJP González, DA Alonso, FML Marcos, D Sánchez-Oliva, IG Ponce

Journal of Human Kinetics, 36, 169-177. http://doi.org/10.2478/hukin-2013-0017

Sánchez-Oliva, D., Leo, F. M., Amado, D., Campos, R. C., \& García-Calvo, T. (2013). Desarrollo y validación del cuestionario de apoyo a las necesidades psicológicas básicas en educación física. Motricidad. European Journal of Human Movement, 30, 53-71.

Sánchez-Oliva, D., Leo, F. M., Amado, D., GonzálezPonce, I., \& García-Calvo, T. (2012). Desarrollo de un cuestionario para valorar la motivación en educación física. Revista Iberoamericana de Psicología del Ejercicio y el Deporte, 7(2), 227-250.

Sanz-Arazuri, E., Ponce-de-León-Elizondo, A., \& Valdemoros-San-Emeterio, M. Á. (2012). Parental predictors of physical inactivity in spanish adolescents. Journal of Sports Science \& Medicine, 11(1), 95-101.

Seabra, A. F., Mendonça, D. M., Thomis, M. A., Anjos, L. A., \& Maia, J. A. (2008). Biological and socio-cultural determinants of physical activity in adolescents. Cadernos de Saúde Pública, 24(4), 721-736. http://doi.org/10.1590/S0102$311 X 2008000400002$

Sheldon, K. M., \& Filak, V. (2008). Manipulating autonomy, competence, and relatedness support in a game-learning context: new evidence that all three needs matter. The British Journal of Social Psychology, 47(Pt 2), 267-283. http://doi.org/10.1348/014466607X238797

Silva, R. B., Matias, T. S., Viana, M. da S., \& Andrade, A. (2012). Relação da prática de exercícios físicos e fatores associados às regulações motivacionais de adolescentes brasileiros. Motricidade, $8(2), \quad 8-21$. http://doi.org/10.6063/motricidade.8(2).708

Skinner, E. A., \& Belmont, M. J. (1993). Motivation in the classroom: Reciprocal effects of teacher behavior and student engagement across the school year. Journal of Educational Psychology, 85(4), 571-581. http://doi.org/10.1037/00220663.85.4.571

Skinner, E. A., \& Edge, K. (2002). Parenting, motivation, and the development of children's coping. Em L. J. Crockett (Ed.), Agency, Motivation, and the Life Course: The Nebraska sym- posium on motivation (pp. 77-143). Lincoln: University of Nebraska Press.

Soenens, B., \& Vansteenkiste, M. (2010). A theoretical upgrade of the concept of parental psychological control: Proposing new insights on the basis of self-determination theory. Developmental Review, 30(1), 74-99. http://doi.org/10.1016/j.dr.2009.11.001

Steinberg, L. (2005). Psychological control: Style or substance? In Changing boundaries of parental authority during adolescence. Em W. Damon \& J. Smetana (Eds.), New directions for child and adolescent development (Vol. 108, pp. 7178). New York: Wiley.

Swaminathan, S., Thomas, T., Yusuf, S., \& Vaz, M. (2013). Clustering of diet, physical activity and overweight in parents and offspring in South India. European Journal of Clinical Nutrition, $67(2)$, $128-134$ http://doi.org/10.1038/ejcn.2012.192

Torregrosa, M., Cruz, J., Sousa, C., Viladrich, C., Villamarín, F., Garcia-Mas, A., \& Palou, P. (2007). La influencia de padres y madres en el compromiso deportivo de futbolistas jóvenes. Revista Latinoamericana de Psicología, 39(2), 227-237.

Wang, C. K. J., \& Biddle, S. J. H. (2001). Young people's motivational profiles in physical activity: A cluster analysis. Journal of Sport and $E_{X}$ ercise Psychology, 23(1), 1-22.

Wilson, K. S., \& Spink, K. S. (2010). Perceived parental social control following a recalled physical activity lapse: Impact on adolescents' reported behavior. Psychology of Sport and Exercise, 11(6), 602-608. http://doi.org/10.1016/j.psychsport.2010.06.0 12

Wuerth, S., Lee, M. J., \& Alfermann, D. (2004). Parental involvement and athletes' career in youth sport. Psychology of Sport and Exercise, 5(1), 21-33. http://doi.org/10.1016/S14690292(02)00047-X

Zhang, T., Solmon, M. A., Kosma, M., Carson, R. L., \& Gu, X. (2011). Need support, need satisfaction, intrinsic motivation, and physical activity participation among middle school students self-determination theory. Journal of Teaching in Physical Education, 30, 51-68. 